\title{
Rewriting biotechnology's story
}

\author{
Michel Morange
}

\author{
Private Science: \\ Biotechnology and the Rise of the \\ Molecular Sciences \\ Edited by Arnold Thackray \\ 1998 University of Pennsylvania Press, \\ 304 pages, $\$ 52.50$ hardcover
}

The explosive development of molecular biotechnologies has already been described in several books such as The Interferon Crusade by Sandra Panem and, more recently, Paul Rabinow's Making PCR. Private Science has a more limited, but more original goal, which appears only after careful reading of its collected essays. In their twelve contributions, the authors try to articulate three different "events": the development of biotechnology, the rise of biomolecular sciences and genetic engineering, and the recent changes in the relationship between public and private science, that is, publicly funded academic research versus the commercial biotechnology industry. Some of the contributions are fully original, while others are short, synthetic presentations of previous studies - such as Robert Bud's contribution, which largely relies on his previous work The Uses of Life: A History of Biotechnology — with a specific emphasis on the events targeted in the book. Some are central to these events, others are more peripheral.

To make a long story short, the authors show that the simplest, and already canonical idea explaining the origins of the modern biotechnology industry-that breakthroughs in molecular biology occurring in academic laboratories allowed the explosive creation of new biotechnology companies in the 1980s (which developed close to the research centers), and abolished the previously clear-cut distinction between public and private research, and universities' and companies' interests-is not entirely valid. For example, the simple scheme of the development of biotechnology companies-with the recruitment of university professors as heads of these new companies-holds only for the United States.

Michel Morange is a professor in the department of biology at the École Normale Supérieure, 46 rue d'Ulm, 75230 Paris Cedex 05, France (morange@wotan.ens.fr). An English translation of his book, A History of Molecular Biology, was recently published by Harvard University Press.

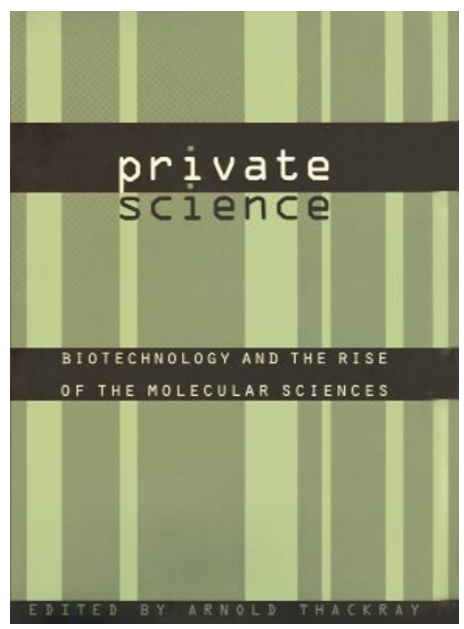

In Britain, for instance, Herbert Gottweis shows that the politics aiming at creating new biotechnology companies led to failure at first, and so molecular biotechnologies simply developed inside large, preexisting pharmaceutical companies. More recently, however, this trend has been reversed, with the UK and the rest of Europe now enjoying governmental and venture capitalist support for biotechnology startups, as well as a change in mindset for academics willing to explore the commercial possibilities of their research.

This is not the only weakness of the canonical vision of the development of molecular biotechnology. In the first chapter, Bud shows us that biotechnology is not new, and later Angela Creager convincingly argues that Edwin Cohn's plasma fractionation project at Harvard Medical School during World War II had already abolished the frontier between academic and private research. The links established between private companies and scientists in the development of the electron microscope, described by Nicolas Rasmussen in Making a Machine Instrumental: RCA and the Wartime Origins of Biological Electron Microscopy in America, 1940-1945-which could have easily found a place in this volume-is another example showing that the changes associated with the development of biotechnology are not new.

Lily Kay argues that molecular biology was, since its birth in the 1930s and long before the rise of genetic engineering techniques, not only a molecular vision of life, but also a biotechnological enterprise with the goal being the "commodification" of molecules and organisms-a "biopower" in the sense used by Michel Foucault, i.e., a power over living organisms, including humans.

These somehow disappointing observations indicate that the distinctions between private and public, and old and new biotechnologies have to be redrawn. New paths in this direction are opened by some of the contributors. One of the characteristics of the new biotechnology is the speed with which information and materials are exchanged. This speed of exchange leads to a permanently moving definition of what is public and what is private.

The careful description of the biotechnology department's new building at Cornell University in New York by Thomas Gieryn demonstrates that the borders between public and private can even run inside the labs themselves. Alberto Cambrosio and Peter Keating argue that the transition between a local and an extended network of relations better describes the rise and stabilization of a new biotechnology than the traditional distinction between academic and private research.

Whatever its new characteristics, biotechnology remains a rich source of "technomyths." Sheldon Krimsky nicely outlines the ambiguous values attached to biotechnology. It has been considered a new and soft technology, more natural compared with historically "environmentally aggressive" chemicalbased technologies. It has also been presented as the new frontier for developed countries, the place of economic competition in the near future. But biotechnology has also been described as a threat to "natural" agriculture-if present agriculture can be called natural-by introducing foreign genes into plants through recombinant methods.

This collection of essays does not allow a single new definition of biotechnology. In fact, many new definitions are possible. It does, however, provide interesting clues to understanding the complex issues associated with the development of the new biotechnologies. My only regret is that it gives more space to the social and economic questions than to the epistemic ones. In particular, the link between the biomolecular revolution and the rise of information technologies is referred to by Kay and Stephen Hilgartner, but not directly addressed. As mentioned by many contributors, the speed of realization is a clear identifier of the "new" biotechnology. As such, this technology fully belongs to our epoch, characterized by the speed of material and information exchange. 